\title{
On Malmquist type theorem of systems of complex difference equations
}

Jianjun Zhang*

\author{
"Correspondence: \\ zhangjianjun1982@163.com \\ Mathematics and Information \\ Technology School, Jiangsu Second \\ Normal University, Nanjing, Jiangsu \\ 210013, P.R. China
}

\begin{abstract}
The main purpose of this paper is to give the Malmquist type result of the meromorphic solutions of a system of complex difference equations of the following form:

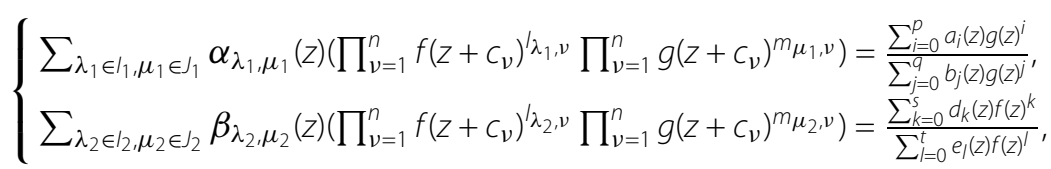

where $c_{1}, c_{2}, \ldots, c_{n}$ are distinct, nonzero complex numbers, the coefficients $\alpha_{\lambda_{1}, \mu_{1}}(z)$ $\left(\lambda_{1} \in I_{1}, \mu_{1} \in J_{1}\right), \beta_{\lambda_{2}, \mu_{2}}(z)\left(\lambda_{2} \in I_{2}, \mu_{2} \in J_{2}\right), a_{i}(z)(i=0,1, \ldots, p), b_{j}(z)(j=0,1, \ldots, q)$, $d_{k}(z)(k=0,1, \ldots, s)$, and $e_{l}(z)(I=0,1, \ldots, t)$ are small functions relative to $f(z)$ and $g(z)$, $I_{i}=\left\{\lambda_{i}=\left.\left(I_{\lambda_{j}, 1}, I_{\lambda_{j}, 2}, \ldots, I_{\lambda_{i, n}, n}\right)\right|_{\lambda_{j, v}} \in N \cup\{0\}, v=1,2, \ldots, n\right\}(i=1,2)$ and $J_{j}=\left\{\mu_{j}=\left(m_{\mu_{j}, 1}, m_{\mu_{j}, 2}, \ldots, m_{\mu_{j}, n}\right) \mid m_{\mu_{j}, v} \in N \cup\{0\}, v=1,2, \ldots, n\right\}(j=1,2)$ are finite index sets. The growth of meromorphic solutions of a related system of complex functional equations is also investigated.
\end{abstract}

Keywords: systems of complex difference equations; meromorphic functions; Malmquist type theorem; functional equation

\section{Introduction and main results}

Let $f(z)$ be a meromorphic function in the complex plane $C$. We assume that the reader is familiar with the standard notations and results in Nevanlinna's value distribution theory of meromorphic functions (see e.g. [1-3]). We use $\rho(f)$ to denote the growth order of a meromorphic function $f(z)$. The notation $S(r, f)$ denotes any quantity that satisfies the condition $S(r, f)=o(T(r, f))$ as $r \rightarrow \infty$ possibly outside an exceptional set of $r$ of finite logarithmic measure. A meromorphic function $a(z)$ is called a small function of $f(z)$ if and only if $T(r, a(z))=S(r, f)$.

In the last ten years, there has been a great deal of interest in studying the properties of complex difference equations (see e.g. [4-20]). Especially, a number of papers (see e.g. $[4,6,10,11,15,17-19])$ focusing on a Malmquist type theorem of the complex difference equations emerged. In 2000, Ablowitz et al. [4] proved some results on the Malmquist theorem of the complex difference equations by utilizing Nevanlinna theory. They obtained the following two results.

(c) 2015 Zhang: licensee Springer. This article is distributed under the terms of the Creative Commons Attribution 4.0 International License (http://creativecommons.org/licenses/by/4.0/), which permits unrestricted use, distribution, and reproduction in any medium, provided you give appropriate credit to the original author(s) and the source, provide a link to the Creative Commons license, and indicate if changes were made. 
Theorem A If the second-order difference equation

$$
f(z+1)+f(z-1)=\frac{a_{0}(z)+a_{1}(z) f+\cdots+a_{p}(z) f^{p}}{b_{0}(z)+b_{1}(z) f+\cdots+b_{q}(z) f^{q}},
$$

with polynomial coefficients $a_{i}(i=1,2, \ldots, p)$ and $b_{j}(j=1,2, \ldots, q)$, admits a transcendental meromorphic solution of finite order, then $d=\max \{p, q\} \leq 2$.

Theorem B If the second-order difference equation

$$
f(z+1) f(z-1)=\frac{a_{0}(z)+a_{1}(z) f+\cdots+a_{p}(z) f^{p}}{b_{0}(z)+b_{1}(z) f+\cdots+b_{q}(z) f^{q}},
$$

with polynomial coefficients $a_{i}(i=1,2, \ldots, p)$ and $b_{j}(j=1,2, \ldots, q)$, admits a transcendental meromorphic solution of finite order, then $d=\max \{p, q\} \leq 2$.

Subsequently, Heittokangas et al. [10], Laine et al. [15] and Huang et al. [11], respectively, gave some generalizations of the above two results. In 2010, the first author in this paper and Liao [18] obtained the following more general result.

Theorem C Let $c_{1}, c_{2}, \ldots, c_{n}$ be distinct, nonzero complex numbers, and suppose that $f(z)$ is a transcendental meromorphic solution of the difference equation

$$
\sum_{\lambda \in I} \alpha_{\lambda}(z)\left(\prod_{\nu=1}^{n} f\left(z+c_{\nu}\right)^{l_{\lambda, \nu}}\right)=\frac{a_{0}(z)+a_{1}(z) f(z)+\cdots+a_{p}(z) f(z)^{p}}{b_{0}(z)+b_{1}(z) f(z)+\cdots+b_{q}(z) f(z)^{q}},
$$

with coefficients $\alpha_{\lambda}(z)(\lambda \in I), a_{i}(z)(i=0,1, \ldots, p)$, and $b_{j}(z)(j=0,1, \ldots, q)$, which are small functions relative to $f(z)$, where $I=\left\{\lambda=\left(l_{\lambda, 1}, l_{\lambda, 2}, \ldots, l_{\lambda, n}\right) \mid l_{\lambda, \nu} \in N \cup\{0\}, \nu=1,2, \ldots, n\right\}$ is a finite index set, and denote

$$
\sigma_{\nu}=\max _{\lambda}\left\{l_{\lambda, \nu}\right\} \quad(v=1,2, \ldots, n), \quad \sigma=\sum_{\nu=1}^{n} \sigma_{\nu}
$$

If the order $\rho(f)$ is finite, then $d=\max \{p, q\} \leq \sigma$.

If all the coefficients in the complex difference equation (1) are rational functions, then in [19], we have the following Malmquist type result, which is reminiscent of the classical Malmquist theorem in complex differential equations.

Theorem $\mathbf{D}$ Let $c_{1}, c_{2}, \ldots, c_{n}$ be distinct, nonzero complex numbers and suppose that $f(z)$ is a transcendental meromorphic solution of the equation

$$
P[z, f]:=\sum_{\lambda \in I} \alpha_{\lambda}(z)\left(\prod_{\nu=1}^{n} f\left(z+c_{\nu}\right)^{l_{\lambda, \nu}}\right)=R(z, f(z))=\frac{P(z, f(z))}{Q(z, f(z))},
$$

where $I=\left\{\lambda=\left(l_{\lambda, 1}, l_{\lambda, 2}, \ldots, l_{\lambda, n}\right) \mid l_{\lambda, v} \in N \cup\{0\}, v=1,2, \ldots, n\right\}$ is a finite index set, $P$ and $Q$ are relatively prime polynomials in $f$ over the field of rational functions, the coefficients $\alpha_{\lambda}$ $(\lambda \in I)$ are rational functions. Denoting the degree of $P[z, f]$ by

$$
\gamma_{P}:=\max _{\lambda \in I}\left\{l_{\lambda, 1}+l_{\lambda, 2}+\cdots+l_{\lambda, n} \mid \lambda=\left(l_{\lambda, 1}, l_{\lambda, 2}, \ldots, l_{\lambda, n}\right)\right\} .
$$


Iff $(z)$ is finite order and has at most finitely many poles, then $R(z, f)$ reduces to a polynomial in $f$ of degree $d \leq \gamma_{P}$.

More recently, people began to study the properties of meromorphic solutions of systems of complex difference equations. In [21], Gao discussed the proximity function and counting function of meromorphic solutions of some classes of systems of complex difference equations. In 2013, Wang et al. [16] investigated the growth of meromorphic solutions of systems of complex difference equations.

Now, we give the Malmquist type result of a system of complex difference equations as follows.

Theorem 1 Let $c_{1}, c_{2}, \ldots, c_{n}$ be distinct, nonzero complex numbers and suppose that $(f(z), g(z))$ is a transcendental meromorphic solution of a system of complex difference equations of the form

$$
\left\{\begin{array}{l}
\sum_{\lambda_{1} \in I_{1}, \mu_{1} \in I_{1}} \alpha_{\lambda_{1}, \mu_{1}}(z)\left(\prod_{\nu=1}^{n} f\left(z+c_{\nu}\right)^{l_{\lambda_{1}, v}} \prod_{\nu=1}^{n} g\left(z+c_{\nu}\right)^{m_{\mu_{1}, v}}\right)=\frac{\sum_{i=0}^{p} a_{i}(z) g(z)^{i}}{\sum_{j=0}^{q} b_{j}(z) g(z)^{j}}, \\
\sum_{\lambda_{2} \in I_{2}, \mu_{2} \in J_{2}} \beta_{\lambda_{2}, \mu_{2}}(z)\left(\prod_{\nu=1}^{n} f\left(z+c_{\nu}\right)^{l_{\lambda_{2}, v}} \prod_{\nu=1}^{n} g\left(z+c_{\nu}\right)^{m_{\mu_{2}, v}}\right)=\frac{\sum_{k=0}^{s} d_{k}(z) f(z)^{k}}{\sum_{l=0}^{t} e_{l}(z) f(z)^{l}}
\end{array}\right.
$$

with coefficients $\alpha_{\lambda_{1}, \mu_{1}}(z)\left(\lambda_{1} \in I_{1}, \mu_{1} \in J_{1}\right), \beta_{\lambda_{2}, \mu_{2}}(z)\left(\lambda_{2} \in I_{2}, \mu_{2} \in J_{2}\right), a_{i}(z)(i=0,1, \ldots, p)$, $b_{j}(z)(j=0,1, \ldots, q), d_{k}(z)(k=0,1, \ldots, s)$, and $e_{l}(z)(l=0,1, \ldots, t)$ are small functions relative to $f(z)$ and $g(z), a_{p}(z), b_{q}(z), d_{s}(z), e_{t}(z) \neq \equiv 0$, where $I_{i}=\left\{\lambda_{i}=\left(l_{\lambda_{i}, 1}, l_{\lambda_{i}, 2}, \ldots, l_{\lambda_{i}, n}\right) \mid l_{\lambda_{i}, v} \in\right.$ $N \cup\{0\}, v=1,2, \ldots, n\}(i=1,2)$, and $J_{j}=\left\{\mu_{j}=\left(m_{\mu_{j}, 1}, m_{\mu_{j}, 2}, \ldots, m_{\mu_{j}, n}\right) \mid m_{\mu_{j}, v} \in N \cup\{0\}, v=\right.$ $1,2, \ldots, n\}(j=1,2)$ are finite index sets, and denote

$$
\begin{array}{ll}
\xi_{1, v}=\max _{\lambda_{1} \in I_{1}}\left\{l_{\lambda_{1}, v}\right\}, & \eta_{1, v}=\max _{\mu_{1} \in I_{1}}\left\{m_{\mu_{1}, v}\right\}, \\
\xi_{2, v}=\max _{\lambda_{2} \in I_{2}}\left\{l_{\lambda_{2}, v}\right\}, & \eta_{2, v}=\max _{\mu_{2} \in J_{2}}\left\{m_{\mu_{2}, v}\right\}
\end{array}
$$

$(v=1,2, \ldots, n)$, and

$$
\sigma_{11}=\sum_{v=1}^{n} \xi_{1, v}, \quad \sigma_{12}=\sum_{v=1}^{n} \eta_{1, v}, \quad \sigma_{21}=\sum_{v=1}^{n} \xi_{2, v}, \quad \sigma_{22}=\sum_{v=1}^{n} \eta_{2, v} .
$$

If $\max \{p, q\}>\sigma_{12}, \max \{s, t\}>\sigma_{21}$, and $\max \{\rho(f), \rho(g)\}<+\infty$, then $\rho(f)=\rho(g)$ and $\left(\max \{p, q\}-\sigma_{12}\right) \cdot\left(\max \{s, t\}-\sigma_{21}\right) \leq \sigma_{11} \sigma_{22}$.

Example 1 It is easy to check that $(f(z), g(z))=(\tan z, \cot z)$ satisfies the following system of difference equations:

$$
\left\{\begin{array}{l}
f\left(z+\frac{\pi}{4}\right) g\left(z+\frac{\pi}{3}\right)^{2}+z g\left(z+\frac{\pi}{4}\right) \\
\quad=\frac{(3 z+1) g^{4}+[(2 \sqrt{3}-6) z+2-2 \sqrt{3}] g^{3}+(4-4 \sqrt{3})(z+1) g^{2}+[(2 \sqrt{3}-2) z+6-2 \sqrt{3}] g+z+3}{3 g^{4}+2 \sqrt{3} g^{3}-2 g^{2}-2 \sqrt{3} g-1} \\
f\left(z+\frac{\pi}{3}\right)^{2} g\left(z+\frac{\pi}{4}\right)+f\left(z+\frac{\pi}{3}\right) g\left(z+\frac{\pi}{4}\right)^{2}=\frac{-(\sqrt{3}+1) f^{4}-2 f^{3}+2 f^{2}-2 f+3+\sqrt{3}}{3 f^{4}+(6-2 \sqrt{3}) f^{3}+(4-4 \sqrt{3}) f^{2}+(2-2 \sqrt{3}) f+1} .
\end{array}\right.
$$

In Example 1, we have $\max \{p, q\}=4, \max \{s, t\}=4, \sigma_{11}=1, \sigma_{12}=3, \sigma_{21}=2, \sigma_{22}=2, \rho(f)=$ $\rho(g)=1<+\infty$, and $\left(\max \{p, q\}-\sigma_{12}\right) \cdot\left(\max \{s, t\}-\sigma_{21}\right)=(4-3)(4-2)=2=1 \times 2=\sigma_{11} \sigma_{22}$. Therefore, the estimation in Theorem 1 is sharp. 
Remark 1 Obviously, if the condition $\max \{p, q\}>\sigma_{12}, \max \{s, t\}>\sigma_{21}$ in Theorem 1 is replaced by $\left(\max \{p, q\}-\sigma_{12}\right)\left(\max \{s, t\}-\sigma_{21}\right)=0$ or $\left(\max \{p, q\}-\sigma_{12}\right)\left(\max \{s, t\}-\sigma_{21}\right)<0$, the estimation $\left(\max \{p, q\}-\sigma_{12}\right) \cdot\left(\max \{s, t\}-\sigma_{21}\right) \leq \sigma_{11} \sigma_{22}$ is still correct. If $\sigma_{11}=0$ or $\sigma_{22}=0$, then the first or second equation in (2) gets the form of (1). For some results as regards (1), the reader may refer to the paper [18].

Remark 2 If the condition $\max \{p, q\}>\sigma_{12}, \max \{s, t\}>\sigma_{21}$ in Theorem 1 is replaced by $\max \{p, q\}<\sigma_{12}, \max \{s, t\}<\sigma_{21}$, then the estimation $\left(\max \{p, q\}-\sigma_{12}\right) \cdot\left(\max \{s, t\}-\sigma_{21}\right) \leq$ $\sigma_{11} \sigma_{22}$ is not true generally. For example, $(f(z), g(z))=(\tan z, \cot z)$ satisfies the following system of complex difference equations:

$$
\left\{\begin{array}{l}
f\left(z+\frac{\pi}{4}\right)^{2} g\left(z-\frac{\pi}{4}\right) g\left(z+\frac{\pi}{4}\right)^{5}+2 g\left(z+\frac{\pi}{4}\right)^{2}=\frac{g^{2}-2 g+1}{g^{2}+2 g+1} \\
f\left(z+\frac{\pi}{4}\right)^{4} f\left(z-\frac{\pi}{4}\right) g\left(z+\frac{\pi}{4}\right)+z f\left(z+\frac{\pi}{4}\right)=\frac{-(z+1) f^{2}-2 f+z+1}{f^{2}-2 f+1}
\end{array}\right.
$$

where $\max \{p, q\}=2, \max \{s, t\}=2, \sigma_{11}=2, \sigma_{12}=6, \sigma_{21}=5, \sigma_{22}=1, \max \{p, q\}<\sigma_{12}$, $\max \{s, t\}<\sigma_{21}$. However, $\left(\max \{p, q\}-\sigma_{12}\right) \cdot\left(\max \{s, t\}-\sigma_{21}\right)=(-4) \times(-3)=12>2=\sigma_{11} \sigma_{22}$.

If $\sigma_{12}=\sigma_{21}=0$, then we have the following simpler result.

Corollary 1 Let $c_{1}, c_{2}, \ldots, c_{n}$ be distinct, nonzero complex numbers, and suppose that $(f(z), g(z))$ is a transcendental meromorphic solution of a system of complex difference equations of the form

$$
\left\{\begin{array}{l}
\sum_{\lambda \in I} \alpha_{\lambda}(z)\left(\prod_{\nu=1}^{n} f\left(z+c_{\nu}\right)^{l_{\lambda, v}}\right)=\frac{\sum_{i=0}^{p} a_{i}(z) g(z)^{i}}{\sum_{j=0}^{q} b_{j}(z) g(z)^{j}}, \\
\sum_{\mu \in J} \beta_{\mu}(z)\left(\prod_{\nu=1}^{n} g\left(z+c_{\nu}\right)^{m_{\mu, \nu}}\right)=\frac{\sum_{k=0}^{s} d_{k}(z) f(z)^{k}}{\sum_{l=0}^{t} e_{l}(z) f(z)^{l}},
\end{array}\right.
$$

with coefficients $\alpha_{\lambda}(z)(\lambda \in I), \beta_{\mu}(z)(\mu \in J), a_{i}(z)(i=0,1, \ldots, p), b_{j}(z)(j=0,1, \ldots, q)$, $d_{k}(z)(k=0,1, \ldots, s)$, and $e_{l}(z)(l=0,1, \ldots, t)$ are small functions relative to $f(z)$ and $g(z)$, $a_{p}(z), b_{q}(z), d_{s}(z), e_{t}(z) \not \equiv 0$, where $I=\left\{\lambda=\left(l_{\lambda, 1}, l_{\lambda, 2}, \ldots, l_{\lambda, n}\right) \mid l_{\lambda, v} \in N \cup\{0\}, v=1,2, \ldots, n\right\}$ and $J=\left\{\mu=\left(m_{\mu, 1}, m_{\mu, 2}, \ldots, m_{\mu, n}\right) \mid m_{\mu, \nu} \in N \cup\{0\}, v=1,2, \ldots, n\right\}$ are two finite index sets, and denote

$$
\xi_{v}=\max _{\lambda}\left\{l_{\lambda, v}\right\} \quad(v=1,2, \ldots, n), \quad \sigma_{1}=\sum_{v=1}^{n} \xi_{v}
$$

and

$$
\eta_{\nu}=\max _{\mu}\left\{m_{\mu, v}\right\} \quad(v=1,2, \ldots, n), \quad \sigma_{2}=\sum_{\nu=1}^{n} \eta_{\nu} .
$$

If $\rho(f)<+\infty$ or $\rho(g)<+\infty$, then $\rho(f)=\rho(g)$ and $\max \{p, q\} \cdot \max \{s, t\} \leq \sigma_{1} \sigma_{2}$.

Example 2 Let $c_{1}=\arctan 2, c_{2}=\arctan (-2)$. It is easy to check that $(f(z), g(z))=(\tan z$, $\cot z$ ) satisfies the following system of difference equations:

$$
\left\{\begin{array}{l}
f\left(z+c_{1}\right)^{2} f\left(z+c_{2}\right)+f\left(z+c_{1}\right) f\left(z+c_{2}\right)^{2}=\frac{-40 g^{3}+10 g}{g^{4}-8 g^{2}+16}, \\
g\left(z+c_{1}\right) g\left(z+c_{2}\right)+g\left(z+c_{1}\right)^{2}=\frac{-20 f^{2}+10 f}{f^{3}+2 f^{2}-4 f-8}
\end{array}\right.
$$


In Example 2, we have $\max \{p, q\}=4, \max \{s, t\}=3, \sigma_{1}=4, \sigma_{2}=3, \rho(f)=\rho(g)=1<$ $+\infty$, and $\max \{p, q\} \cdot \max \{s, t\}=\sigma_{1} \cdot \sigma_{2}=12$. Therefore, the estimation in Corollary 1 is sharp.

In [15], Laine et al. also considered the growth of meromorphic solutions of some classes of complex difference functional equations and obtained the following result.

Theorem E Suppose that $f$ is a transcendental meromorphic solution of the equation

$$
\sum_{\{\}} \alpha_{J}(z)\left(\prod_{j \in J} f\left(z+c_{j}\right)\right)=f(p(z))
$$

where $p(z)$ is a polynomial of degree $k \geq 2,\{J\}$ is the collection of all subsets of $\{1,2, \ldots, n\}$. Moreover, we assume that the coefficients $\alpha_{J}(z)$ are small functions relative to $f$ and that $n \geq k$. Then

$$
T(r, f)=O\left((\log r)^{\alpha+\varepsilon}\right)
$$

where $\alpha=\frac{\log n}{\log k}$.

In 2010, Zhang et al. [18] got a more generalized result than Theorem E. Next we give the growth of meromorphic solutions of a system of complex functional equations as follows.

Theorem 2 Let $c_{1}, c_{2}, \ldots, c_{n}$ be distinct, nonzero complex numbers and suppose that $(f(z), g(z))$ is a transcendental meromorphic solution of a system of complex functional equations of the form

$$
\left\{\begin{array}{l}
\sum_{\lambda_{1} \in I_{1}, \mu_{1} \in J_{1}} \alpha_{\lambda_{1}, \mu_{1}}(z)\left(\prod_{\nu=1}^{n} f\left(z+c_{\nu}\right)^{l_{\lambda_{1}, v}} \prod_{\nu=1}^{n} g\left(z+c_{v}\right)^{m_{\mu_{1}, v}}\right)=f(p(z)), \\
\sum_{\lambda_{2} \in I_{2}, \mu_{2} \in J_{2}} \beta_{\lambda_{2}, \mu_{2}}(z)\left(\prod_{\nu=1}^{n} f\left(z+c_{\nu}\right)^{l_{\lambda_{2}, v}} \prod_{\nu=1}^{n} g\left(z+c_{\nu}\right)^{m_{\mu_{2}, v}}\right)=g(p(z)),
\end{array}\right.
$$

where $p(z)$ is a polynomial of degree $k \geq 2, I_{i}=\left\{\lambda_{i}=\left(l_{\lambda_{i}, 1}, l_{\lambda_{i}, 2}, \ldots, l_{\lambda_{i}, n}\right) \mid l_{\lambda_{i}, v} \in N \cup\{0\}, v=\right.$ $1,2, \ldots, n\}(i=1,2)$ and $J_{j}=\left\{\mu_{j}=\left(m_{\mu_{j}, 1}, m_{\mu_{j}, 2}, \ldots, m_{\mu_{j}, n}\right) \mid m_{\mu_{j}, v} \in N \cup\{0\}, v=1,2, \ldots, n\right\}(j=$ $1,2)$ are finite index sets, and denote

$$
\begin{array}{ll}
\xi_{1, v}=\max _{\lambda_{1} \in I_{1}}\left\{l_{\lambda_{1}, v}\right\}, & \eta_{1, v}=\max _{\mu_{1} \in J_{1}}\left\{m_{\mu_{1}, v}\right\}, \\
\xi_{2, v}=\max _{\lambda_{2} \in I_{2}}\left\{l_{\lambda_{2}, v}\right\}, & \eta_{2, v}=\max _{\mu_{2} \in J_{2}}\left\{m_{\mu_{2}, v}\right\}
\end{array}
$$

$(v=1,2, \ldots, n)$,

$$
\sigma_{11}=\sum_{\nu=1}^{n} \xi_{1, v}, \quad \sigma_{12}=\sum_{\nu=1}^{n} \eta_{1, v}, \quad \sigma_{21}=\sum_{\nu=1}^{n} \xi_{2, v}, \quad \sigma_{22}=\sum_{\nu=1}^{n} \eta_{2, v}
$$

and

$$
\sigma=\max \left\{\sigma_{11}, \sigma_{12}, \sigma_{21}, \sigma_{22}\right\}
$$


Moreover, we assume that the coefficients $\alpha_{\lambda_{1}, \mu_{1}}(z)\left(\lambda_{1} \in I_{1}, \mu_{1} \in J_{1}\right), \beta_{\lambda_{2}, \mu_{2}}(z)\left(\lambda_{2} \in I_{2}, \mu_{2} \in\right.$ $J_{2}$ ) are small functions relative to $f(z)$ and $g(z)$, and that $2 \sigma \geq k$. Then

$$
T(r, f)=O\left((\log r)^{\alpha+\varepsilon}\right), \quad T(r, g)=O\left((\log r)^{\alpha+\varepsilon}\right),
$$

where $\alpha=\frac{\log 2 \sigma}{\log k}$.

If $\sigma_{12}=\sigma_{21}=0$, then we can obtain the following result easily.

Corollary 2 Let $c_{1}, c_{2}, \ldots, c_{n}$ be distinct, nonzero complex numbers and suppose that $(f(z), g(z))$ is a transcendental meromorphic solution of a system of complex functional equations of the form

$$
\left\{\begin{array}{l}
\sum_{\lambda \in I} \alpha_{\lambda}(z)\left(\prod_{v=1}^{n} f\left(z+c_{v}\right)^{l_{\lambda, v}}\right)=g(p(z)), \\
\sum_{\mu \in J} \beta_{\mu}(z)\left(\prod_{v=1}^{n} g\left(z+c_{v}\right)^{m_{\mu, v}}\right)=f(p(z)),
\end{array}\right.
$$

where $p(z)$ is a polynomial of degree $k \geq 2, I=\left\{\lambda=\left(l_{\lambda, 1}, l_{\lambda, 2}, \ldots, l_{\lambda, n}\right) \mid l_{\lambda, \nu} \in N \cup\{0\}, v=\right.$ $1,2, \ldots, n\}$ and $J=\left\{\mu=\left(m_{\mu, 1}, m_{\mu, 2}, \ldots, m_{\mu, n}\right) \mid m_{\mu, \nu} \in N \cup\{0\}, v=1,2, \ldots, n\right\}$ are two finite index sets, and denote

$$
\begin{array}{ll}
\xi_{v}=\max _{\lambda}\left\{l_{\lambda, v}\right\} \quad(v=1,2, \ldots, n), & \sigma_{1}=\sum_{v=1}^{n} \xi_{v}, \\
\eta_{v}=\max _{\mu}\left\{m_{\mu, v}\right\} \quad(v=1,2, \ldots, n), & \sigma_{2}=\sum_{\nu=1}^{n} \eta_{v}
\end{array}
$$

and

$$
\sigma=\max \left\{\sigma_{1}, \sigma_{2}\right\} .
$$

Moreover, we assume that the coefficients $\alpha_{\lambda}(z)(\lambda \in I), \beta_{\mu}(z)(\mu \in J)$ are small functions relative to $f(z)$ and $g(z)$, and that $2 \sigma \geq k$. Then

$$
T(r, f)=O\left((\log r)^{\alpha+\varepsilon}\right), \quad T(r, g)=O\left((\log r)^{\alpha+\varepsilon}\right),
$$

where $\alpha=\frac{\log 2 \sigma}{\log k}$.

\section{Some lemmas}

In order to prove our results, we need the following lemmas.

Lemma 1 (see [3]) Let $f(z)$ be a meromorphic function. Then for all irreducible rational functions inf,

$$
R(z, f)=\frac{P(z, f)}{Q(z, f)}=\frac{\sum_{i=0}^{p} a_{i}(z) f^{i}}{\sum_{j=0}^{q} b_{j}(z) f^{j}}
$$

such that the meromorphic coefficients $a_{i}(z), b_{j}(z)$ satisfy

$$
\begin{cases}T\left(r, a_{i}\right)=S(r, f), & i=0,1, \ldots, p, \\ T\left(r, b_{j}\right)=S(r, f), & j=0,1, \ldots, q,\end{cases}
$$


we have

$$
T(r, R(z, f))=\max \{p, q\} \cdot T(r, f)+S(r, f) .
$$

In [22], AZ Mokhon'ko and VD Mokhon'ko gave an estimation of Nevanlinna's characteristic function of

$$
F(z)=\frac{P(z)}{Q(z)}=\frac{\sum_{\lambda \in I} f_{1,1}^{l_{\lambda, 1}} f_{2}^{l_{\lambda, 2}} \cdots f_{n}^{l_{\lambda, n}}}{\sum_{\mu \in J} f_{1}^{m_{\mu, 1}} f_{2}^{m_{\mu, 2}} \cdots f_{n}^{m_{\mu, n}}},
$$

where $f_{1}, f_{2}, \ldots, f_{n}$ are distinct meromorphic functions, $I=\left\{\lambda=\left(l_{\lambda, 1}, l_{\lambda, 2}, \ldots, l_{\lambda, n}\right) \mid l_{\lambda, v} \in\right.$ $N \cup\{0\}, v=1,2, \ldots, n\}$ and $J=\left\{\mu=\left(m_{\mu, 1}, m_{\mu, 2}, \ldots, m_{\mu, n}\right) \mid m_{\mu, \nu} \in N \cup\{0\}, v=1,2, \ldots, n\right\}$ are two finite index sets. However, the method of the proof was too complex. For $F(z)$ of the form $\sum_{\lambda \in I} f_{1}^{l_{\lambda, 1}} f_{2}^{l_{\lambda, 2}} \cdots f_{n}^{l_{\lambda, n}}$, Zheng et al. [20] gave a simpler proof, but the estimation of $T(r, F)$ was not sharp. For completeness, we give the proof of the following lemma.

Lemma 2 Let $f_{1}, f_{2}, \ldots, f_{n}$ be distinct meromorphic functions. Then

$$
T\left(r, \sum_{\lambda \in I} f_{1}^{l_{\lambda, 1}} f_{2}^{l_{\lambda, 2}} \cdots f_{n}^{l_{\lambda, n}}\right) \leq \sum_{j=1}^{n} \sigma_{j} T\left(r, f_{j}\right)+\log t,
$$

where $I=\left\{\left(l_{\lambda, 1}, l_{\lambda, 2}, \ldots, l_{\lambda, n}\right) \mid l_{\lambda, j} \in N \cup\{0\}, j=1,2, \ldots, n\right\}$ is an finite index set consisting of $t$ elements and $\sigma_{j}=\max _{\lambda \in I}\left\{l_{\lambda, j}\right\}(j=1,2, \ldots, n)$.

Proof All the poles of the function $\sum_{\lambda \in I} f_{1}^{l_{\lambda, 1}} f_{2}^{l_{\lambda, 2}} \cdots f_{n}^{l_{\lambda, n}}$ are generated by the poles of the functions $f_{j}(j=1,2, \ldots, n)$, and every pole of multiplicity $k$ of $f_{j}(j=1,2, \ldots, n)$ has order at most $k \sigma_{j}$. This implies that

$$
n\left(r, \sum_{\lambda \in I} f_{1}^{l_{\lambda, 1}} f_{2}^{l_{\lambda, 2}} \cdots f_{n}^{l_{\lambda, n}}\right) \leq \sum_{j=1}^{n} \sigma_{j} n\left(r, f_{j}\right) .
$$

Thus we obtain

$$
N\left(r, \sum_{\lambda \in I} f_{1}^{l_{\lambda, 1}} f_{2}^{l_{\lambda, 2}} \cdots f_{n}^{l_{\lambda, n}}\right) \leq \sum_{j=1}^{n} \sigma_{j} N\left(r, f_{j}\right) .
$$

We next prove that

$$
m\left(r, \sum_{\lambda \in I} f_{1}^{l_{\lambda, 1}} f_{2}^{l_{\lambda, 2}} \cdots f_{n}^{l_{\lambda, n}}\right) \leq \sum_{j=1}^{n} \sigma_{j} m\left(r, f_{j}\right)+\log t
$$

and we define

$$
\begin{cases}f_{j}^{*}(z)=f_{j}(z), & \left|f_{j}(z)\right|>1 \\ f_{j}^{*}(z)=1, & \left|f_{j}(z)\right| \leq 1\end{cases}
$$


for $j=1,2, \ldots, n$. Thus we have

$$
\begin{aligned}
\left|\sum_{\lambda \in I} f_{1}^{l_{\lambda, 1}} f_{2}^{l_{\lambda, 2}} \cdots f_{n}^{l_{\lambda, n}}\right| & \leq \sum_{\lambda \in I}\left|f_{1}^{l_{\lambda, 1}} f_{2}^{l_{\lambda, 2}} \cdots f_{n}^{l_{\lambda, n}}\right| \\
& \leq \sum_{\lambda \in I}\left|f_{1}^{* l_{\lambda, 1}} f_{2}^{* l_{\lambda, 2}} \cdots f_{n}^{* l_{\lambda, n}}\right| \\
& =\left|f_{1}^{* \sigma_{1}} f_{2}^{* \sigma_{2}} \cdots f_{n}^{* \sigma_{n}}\right|\left(\sum_{\lambda \in I} \frac{\left|f_{1}^{* l_{\lambda, 1}} f_{2}^{* l_{\lambda, 2}} \cdots f_{n}^{* l_{\lambda, n}}\right|}{\left|f_{1}^{* \sigma_{1}} f_{2}^{* \sigma_{2}} \cdots f_{n}^{* \sigma_{n}}\right|}\right) \\
& \leq t\left|f_{1}^{* \sigma_{1}} f_{2}^{* \sigma_{2}} \cdots f_{n}^{* \sigma_{n}}\right| .
\end{aligned}
$$

By the definition of $m(r, f)$, we immediately conclude that

$$
\begin{aligned}
m\left(r, \sum_{\lambda \in I} f_{1}^{l_{\lambda, 1}} f_{2}^{l_{\lambda, 2}} \cdots f_{n}^{l_{\lambda, n}}\right) & =\frac{1}{2 \pi} \int_{0}^{2 \pi} \log ^{+}\left|\sum_{\lambda \in I} f_{1}^{l_{\lambda, 1}} f_{2}^{l_{\lambda, 2}} \cdots f_{n}^{l_{\lambda, n}}\right| d \theta \\
& \leq \frac{1}{2 \pi} \int_{0}^{2 \pi} \log ^{+}\left|f_{1}^{* \sigma_{1}} f_{2}^{* \sigma_{2}} \cdots f_{n}^{* \sigma_{n}}\right| d \theta+\log t \\
& =\sum_{j=1}^{n} \sigma_{j} m\left(r, f_{j}\right)+\log t .
\end{aligned}
$$

By (7) and (8), the assertion follows.

Remark 3 If we suppose that $\alpha_{\lambda}(z)=o\left(T\left(r, f_{j}\right)\right)(\lambda \in I)$ hold for all $j \in\{1,2, \ldots, n\}$, and denote $T\left(r, a_{\lambda}\right)=S(r, f)(\lambda \in I)$, then we have the following estimation:

$$
T\left(r, \sum_{\lambda \in I} \alpha_{\lambda}(z) f_{1}^{l_{\lambda, 1}} f_{2}^{l_{\lambda, 2}} \cdots f_{n}^{l_{\lambda, n}}\right) \leq \sum_{j=1}^{n} \sigma_{j} T\left(r, f_{j}\right)+S(r, f) .
$$

Lemma 3 (see [6]) Let $f(z)$ be a meromorphic function with order $\rho=\rho(f), \rho<+\infty$, and $c$ be a fixed non zero complex number, then for each $\varepsilon>0$, we have

$$
T(r, f(z+c))=T(r, f)+O\left(r^{\rho-1+\varepsilon}\right)+O(\log r) .
$$

Lemma 4 (see [3]) Let $g:(0,+\infty) \rightarrow R, h:(0,+\infty) \rightarrow R$ be monotone increasing functions such that $g(r) \leq h(r)$ outside of an exceptional set $E$ of finite linear measure. Then, for any $\alpha>1$, there exists $r_{0}>0$ such that $g(r) \leq h(\alpha r)$ for all $r_{0}$.

Lemma 5 (see [23]) Let $f$ be a transcendental meromorphic function, and $p(z)=a_{k} z^{k}+$ $a_{k-1} z^{k-1}+\cdots+a_{1} z+a_{0}, a_{k} \neq 0$, be a nonconstant polynomial of degree $k$. Given $0<\delta<\left|a_{k}\right|$, denote $\lambda=\left|a_{k}\right|+\delta$ and $\mu=\left|a_{k}\right|-\delta$. Then given $\varepsilon>0$ and $a \in C \cup\{\infty\}$, we have

$$
\begin{aligned}
& k n\left(\mu r^{k}, a, f\right) \leq n(r, a, f(p(z))) \leq k n\left(\lambda r^{k}, a, f\right), \\
& N\left(\mu r^{k}, a, f\right)+O(\log r) \leq N(r, a, f(p(z))) \leq N\left(\lambda r^{k}, a, f\right)+O(\log r), \\
& (1-\varepsilon) T\left(\mu r^{k}, f\right) \leq T(r, f(p(z))) \leq(1+\varepsilon) T\left(\lambda r^{k}, f\right),
\end{aligned}
$$

for all r large enough. 


\section{Proofs of theorems}

Proof of Theorem 1 We assume that $(f(z), g(z))$ is a transcendental meromorphic solution of the system of complex difference equations (2). By the first equation in (2), Lemma 1, Lemma 2, and Lemma 3, we have, for each $\varepsilon>0$,

$$
\begin{aligned}
& \max \{p, q\} T(r, g) \\
&=T\left(r, \sum_{\lambda_{1} \in I_{1}, \mu_{1} \in J_{1}} \alpha_{\lambda_{1}, \mu_{1}}(z)\left(\prod_{\nu=1}^{n} f\left(z+c_{\nu}\right)^{l_{\lambda_{1}, v}} \prod_{\nu=1}^{n} g\left(z+c_{\nu}\right)^{m_{\mu_{1}, v}}\right)\right)+S(r, g) \\
& \leq \sum_{\nu=1}^{n} \xi_{1, v} T\left(r, f\left(z+c_{\nu}\right)\right)+\sum_{\nu=1}^{n} \eta_{1, v} T\left(r, g\left(z+c_{v}\right)\right)+S(r, f)+S(r, g) \\
&=\sum_{\nu=1}^{n} \xi_{1, v} T(r, f(z))+O\left(r^{\rho(f)-1+\varepsilon}\right)+\sum_{v=1}^{n} \eta_{1, v} T(r, g(z))+O\left(r^{\rho(g)-1+\varepsilon}\right) \\
&+O(\log r)+S(r, f)+S(r, g) \\
&=\left(\sum_{\nu=1}^{n} \xi_{1, v}\right) T(r, f(z))+\left(\sum_{v=1}^{n} \eta_{1, v}\right) T(r, g(z)) \\
&+O\left(r^{\rho(g)-1+\varepsilon}\right)+O(\log r)+S(r, f)+S(r, g) \\
&= \sigma_{11} T(r, f(z))+\sigma_{12} T(r, g(z))+O\left(r^{\rho(f)-1+\varepsilon}\right)+O\left(r^{\rho(g)-1+\varepsilon}\right) \\
&+O(\log r)+S(r, f)+S(r, g) .
\end{aligned}
$$

By the above inequality, we get, for each $\varepsilon>0$,

$$
\begin{aligned}
\left(\max \{p, q\}-\sigma_{12}\right) T(r, g) \\
\leq \sigma_{11} T(r, f(z))+O\left(r^{\rho(f)-1+\varepsilon}\right)+O\left(r^{\rho(g)-1+\varepsilon}\right) \\
\quad+O(\log r)+S(r, f)+S(r, g) .
\end{aligned}
$$

Since $\max \{p, q\}>\sigma_{12}$ by the assumption, we have, for each $\varepsilon>0$,

$$
\begin{aligned}
T(r, g) \leq & \frac{\sigma_{11}}{\max \{p, q\}-\sigma_{12}} T(r, f(z))+O\left(r^{\rho(f)-1+\varepsilon}\right)+O\left(r^{\rho(g)-1+\varepsilon}\right) \\
& +O(\log r)+S(r, f)+S(r, g) .
\end{aligned}
$$

Similarly, by the second equation in (2), we obtain, for each $\varepsilon>0$,

$$
\begin{aligned}
\max & \{s, t\} T(r, f) \\
& =T\left(r, \sum_{\lambda_{2} \in I_{2}, \mu_{2} \in J_{2}} \beta_{\lambda_{2}, \mu_{2}}(z)\left(\prod_{\nu=1}^{n} f\left(z+c_{\nu}\right)^{l_{\lambda_{2}, v}} \prod_{\nu=1}^{n} g\left(z+c_{\nu}\right)^{m_{\mu_{2}, \nu}}\right)\right)+S(r, f) \\
& \leq \sum_{\nu=1}^{n} \xi_{2, v} T\left(r, f\left(z+c_{v}\right)\right)+\sum_{\nu=1}^{n} \eta_{2, v} T\left(r, g\left(z+c_{\nu}\right)\right)+S(r, f)+S(r, g) \\
& =\sum_{\nu=1}^{n} \xi_{2, v} T(r, f(z))+O\left(r^{\rho(f)-1+\varepsilon}\right)+\sum_{\nu=1}^{n} \eta_{2, v} T(r, g(z))
\end{aligned}
$$




$$
\begin{aligned}
& +O\left(r^{\rho(g)-1+\varepsilon}\right)+O(\log r)+S(r, f)+S(r, g) \\
= & \left(\sum_{v=1}^{n} \xi_{2, v}\right) T(r, f(z))+\left(\sum_{v=1}^{n} \eta_{2, v}\right) T(r, g(z)) \\
& +O(\log r)+S(r, f)+S(r, g) \\
= & \sigma_{21} T(r, f(z))+\sigma_{22} T(r, g(z))+O\left(r^{\rho(f)-1+\varepsilon}\right)+O\left(r^{\rho(g)-1+\varepsilon}\right) \\
& +O(\log r)+S(r, f)+S(r, g) .
\end{aligned}
$$

By (12) and $\max \{s, t\}>\sigma_{21}$, we have, for each $\varepsilon>0$,

$$
\begin{aligned}
\left(\max \{s, t\}-\sigma_{21}\right) T(r, f) \\
\leq \sigma_{22} T(r, g(z))+O\left(r^{\rho(f)-1+\varepsilon}\right)+O\left(r^{\rho(g)-1+\varepsilon}\right) \\
\quad+O(\log r)+S(r, f)+S(r, g)
\end{aligned}
$$

and

$$
\begin{aligned}
T(r, f) \leq & \frac{\sigma_{22}}{\max \{s, t\}-\sigma_{21}} T(r, g(z))+O\left(r^{\rho(f)-1+\varepsilon}\right)+O\left(r^{\rho(g)-1+\varepsilon}\right) \\
& +O(\log r)+S(r, f)+S(r, g) .
\end{aligned}
$$

Using (11), we can obtain $\rho(g) \leq \rho(f)$. Similarly, we can get $\rho(f) \leq \rho(g)$ from (14). Therefore, we have $\rho(f)=\rho(g)$.

It follows from (10) and (13) that

$$
\begin{aligned}
& \left(\max \{p, q\}-\sigma_{12}\right)\left(\max \{s, t\}-\sigma_{21}\right) T(r, f) T(r, g) \\
& \quad \leq \sigma_{11} \sigma_{22} T(r, f(z)) T(r, g(z))+o(T(r, f) T(r, g)) .
\end{aligned}
$$

From (15), we conclude that

$$
\left(\max \{p, q\}-\sigma_{12}\right) \cdot\left(\max \{s, t\}-\sigma_{21}\right) \leq \sigma_{11} \sigma_{22} .
$$

This yields the asserted result.

Proof of Theorem 2 We assume that $(f(z), g(z))$ is a transcendental meromorphic solution of a system of complex functional equations (5). Let $C=\max \left\{\left|c_{1}\right|,\left|c_{2}\right|, \ldots,\left|c_{n}\right|\right\}$. According to the first equation in (5), Lemma 2, Lemma 3 , and the last assertion of Lemma 5, we get

$$
\begin{aligned}
(1 & -\varepsilon) T\left(\mu r^{k}, f\right) \\
& \leq T(r, f(p(z))) \\
& =T\left(r, \sum_{\lambda_{1} \in I_{1}, \mu_{1} \in J_{1}} \alpha_{\lambda_{1}, \mu_{1}}(z)\left(\prod_{\nu=1}^{n} f\left(z+c_{\nu}\right)^{l_{\lambda_{1}, v}} \prod_{\nu=1}^{n} g\left(z+c_{v}\right)^{m_{\mu_{1}, v}}\right)\right) \\
& \leq \sum_{\nu=1}^{n} \xi_{1, v} T\left(r, f\left(z+c_{v}\right)\right)+\sum_{\nu=1}^{n} \eta_{1, v} T\left(r, g\left(z+c_{v}\right)\right)+S(r, f)+S(r, g)
\end{aligned}
$$




$$
\begin{aligned}
& \leq \sum_{\nu=1}^{n} \xi_{1, v} T(r+C, f(z))+\sum_{v=1}^{n} \eta_{1, v} T(r+C, g(z))+S(r, f)+S(r, g) \\
& =\left(\sum_{\nu=1}^{n} \xi_{1, v}\right) T(r+C, f(z))+\left(\sum_{v=1}^{n} \eta_{1, v}\right) T(r+C, g(z))+S(r, f)+S(r, g) \\
& =\sigma_{11} T(r+C, f(z))+\sigma_{12} T(r+C, g(z))+S(r, f)+S(r, g) .
\end{aligned}
$$

Since $T(r+C, f) \leq T(\beta r, f)$ and $T(r+C, g) \leq T(\beta r, g)$ hold for $r$ large enough for $\beta>1$, we may assume $r$ to be large enough to satisfy

$$
(1-\varepsilon) T\left(\mu r^{k}, f\right) \leq \sigma_{11}(1+\varepsilon) T(\beta r, f)+\sigma_{12}(1+\varepsilon) T(\beta r, g)
$$

outside a possible exceptional set of finite linear measure. By Lemma 4, we know that, whenever $\gamma>1$,

$$
(1-\varepsilon) T\left(\mu r^{k}, f\right) \leq \sigma_{11}(1+\varepsilon) T(\gamma \beta r, f)+\sigma_{12}(1+\varepsilon) T(\gamma \beta r, g)
$$

holds for all $r$ large enough. Let $t=\gamma \beta r$, then the inequality (16) may be written in the form

$$
T\left(\frac{\mu}{(\gamma \beta)^{k}} t^{k}, f\right) \leq \frac{\sigma_{11}(1+\varepsilon)}{1-\varepsilon} T(t, f)+\frac{\sigma_{12}(1+\varepsilon)}{1-\varepsilon} T(t, g)
$$

Similarly, by the second equation in (5), for all $r$ large enough and $\beta>1, \gamma>1$, we have

$$
\begin{aligned}
(1 & -\varepsilon) T\left(\mu r^{k}, g\right) \\
& \leq T(r, g(p(z))) \\
& =T\left(r, \sum_{\lambda_{2} \in I_{2}, \mu_{2} \in I_{2}} \beta_{\lambda_{2}, \mu_{2}}(z)\left(\prod_{\nu=1}^{n} f\left(z+c_{\nu}\right)^{l_{2}, v} \prod_{\nu=1}^{n} g\left(z+c_{v}\right)^{m_{\mu_{2}, v}}\right)\right) \\
& \leq \sum_{\nu=1}^{n} \xi_{2, v} T\left(r, f\left(z+c_{v}\right)\right)+\sum_{\nu=1}^{n} \eta_{2, v} T\left(r, g\left(z+c_{v}\right)\right)+S(r, f)+S(r, g) \\
& \leq \sum_{\nu=1}^{n} \xi_{2, v} T(r+C, f(z))+\sum_{v=1}^{n} \eta_{2, v} T(r+C, g(z))+S(r, f)+S(r, g) \\
& =\left(\sum_{\nu=1}^{n} \xi_{2, v}\right) T(r+C, f(z))+\left(\sum_{\nu=1}^{n} \eta_{2, v}\right) T(r+C, g(z))+S(r, f)+S(r, g) \\
& =\sigma_{21} T(r+C, f(z))+\sigma_{22} T(r+C, g(z))+S(r, f)+S(r, g) \\
& \leq \sigma_{21}(1+\varepsilon) T(\gamma \beta r, f)+\sigma_{22}(1+\varepsilon) T(\gamma \beta r, g) .
\end{aligned}
$$

Let $t=\gamma \beta r$, we have

$$
T\left(\frac{\mu}{(\gamma \beta)^{k}} t^{k}, g\right) \leq \frac{\sigma_{21}(1+\varepsilon)}{1-\varepsilon} T(t, f)+\frac{\sigma_{22}(1+\varepsilon)}{1-\varepsilon} T(t, g) .
$$


Letting $s=\log t+\frac{\log \frac{\mu}{(\gamma \beta)^{k}}}{k-1}$, then $t=e^{s}\left(\frac{\mu}{(\gamma \beta)^{k}}\right)^{\frac{1}{1-k}}$ and

$$
\begin{aligned}
k s & =k \log t+\frac{k}{k-1} \log \frac{\mu}{(\gamma \beta)^{k}} \\
& =k \log t+\log \frac{\mu}{(\gamma \beta)^{k}}+\frac{\log \frac{\mu}{(\gamma \beta)^{k}}}{k-1} \\
& =\log \frac{\mu}{(\gamma \beta)^{k}} t^{k}+\log \left(\frac{\mu}{(\gamma \beta)^{k}}\right)^{\frac{1}{k-1}} .
\end{aligned}
$$

So

$$
\frac{\mu}{(\gamma \beta)^{k}} t^{k}=e^{k s}\left(\frac{\mu}{(\gamma \beta)^{k}}\right)^{\frac{1}{1-k}} .
$$

Let $T(t, f)=T\left(e^{s}\left(\frac{\mu}{(\gamma \beta)^{k}}\right)^{\frac{1}{1-k}}, f\right)=\Phi(s, f), T(t, g)=T\left(e^{s}\left(\frac{\mu}{(\gamma \beta)^{k}}\right)^{\frac{1}{1-k}}, g\right)=\Phi(s, g), \quad M=$ $\max \left\{\frac{\sigma_{11}(1+\varepsilon)}{1-\varepsilon}, \frac{\sigma_{12}(1+\varepsilon)}{1-\varepsilon}, \frac{\sigma_{21}(1+\varepsilon)}{1-\varepsilon}, \frac{\sigma_{22}(1+\varepsilon)}{1-\varepsilon}\right\}$, then from (17) and (19), we have

$$
\begin{aligned}
\Phi(k s, f) & =T\left(e^{k s}\left(\frac{\mu}{(\gamma \beta)^{k}}\right)^{\frac{1}{1-k}}, f\right) \\
& =T\left(\frac{\mu}{(\gamma \beta)^{k}} t^{k}, f\right) \\
& \leq \frac{\sigma_{11}(1+\varepsilon)}{1-\varepsilon} T(t, f)+\frac{\sigma_{12}(1+\varepsilon)}{1-\varepsilon} T(t, g) \\
& \leq M \Phi(s, f)+M \Phi(s, g) .
\end{aligned}
$$

Similarly, from (18), we can get

$$
\Phi(k s, g) \leq M \Phi(s, f)+M \Phi(s, g)
$$

The inequalities (20) and (21) hold for all $s$ large enough.

Letting now $\alpha=\frac{\log 2 M}{\log k}$, namely, $2 M=k^{\alpha}$. Write $\Psi(s, f)=\frac{\Phi(s, f)}{s^{\alpha}}$ and $\Psi(s, g)=\frac{\Phi(s, g)}{s^{\alpha}}$, thus we have

$$
\Psi(k s, f) \leq \frac{1}{2} \Psi(s, f)+\frac{1}{2} \Psi(s, g)
$$

and

$$
\Psi(k s, g) \leq \frac{1}{2} \Psi(s, f)+\frac{1}{2} \Psi(s, g) .
$$

The inequalities (22) and (23) hold for all $s$ large enough, we may assume that (22) and (23) hold for all $s \geq s_{0}$.

Let $M_{1}=\sup _{s_{0} \leq s \leq k s_{0}} \Psi(s, f)$ and $M_{2}=\sup _{s_{0} \leq s \leq k s_{0}} \Psi(s, g)$, then by (22) and (23) we can obtain

$$
\begin{aligned}
& \sup _{s \in\left[k s_{0}, k^{2} s_{0}\right]} \Psi(s, f)=\sup _{s \in\left[s_{0}, k s_{0}\right]} \Psi(k s, f) \leq \frac{1}{2} \sup _{s \in\left[s_{0}, k s_{0}\right]} \Psi(s, f)+\frac{1}{2} \sup _{s \in\left[s_{0}, k s_{0}\right]} \Psi(s, g) \leq \frac{M_{1}}{2}+\frac{M_{2}}{2}, \\
& \sup _{s \in\left[k s_{0}, k^{2} s_{0}\right]} \Psi(s, g)=\sup _{s \in\left[s_{0}, k s_{0}\right]} \Psi(k s, g) \leq \frac{1}{2} \sup _{s \in\left[s_{0}, k s_{0}\right]} \Psi(s, f)+\frac{1}{2} \sup _{s \in\left[s_{0}, k s_{0}\right]} \Psi(s, g) \leq \frac{M_{1}}{2}+\frac{M_{2}}{2} .
\end{aligned}
$$


Similarly, we have

$$
\begin{aligned}
& \sup _{s \in\left[k^{2} s_{0}, k^{3} s_{0}\right]} \Psi(s, f)=\sup _{s \in\left[k s_{0}, k^{2} s_{0}\right]} \Psi(k s, f) \leq \frac{1}{2} M_{1}+\frac{1}{2} M_{2}, \\
& \sup _{s \in\left[k^{2} s_{0}, k^{3} s_{0}\right]} \Psi(s, g)=\sup _{s \in\left[k s_{0}, k^{2} s_{0}\right]} \Psi(k s, g) \leq \frac{1}{2} M_{1}+\frac{1}{2} M_{2},
\end{aligned}
$$

Thus, we deduce that

$$
\begin{aligned}
& \sup _{s \geq k s_{0}} \Psi(s, f) \leq \frac{1}{2} M_{1}+\frac{1}{2} M_{2}<+\infty, \\
& \sup _{s \geq k s_{0}} \Psi(s, g) \leq \frac{1}{2} M_{1}+\frac{1}{2} M_{2}<+\infty .
\end{aligned}
$$

Therefore, $\Psi(s, f)$ and $\Psi(s, g)$ are bounded for all $s \geq s_{0}$. There exist some constants $K_{1}$, $K_{2}, K_{3}, K_{4}$, such that, for any $\varepsilon>0$,

$$
T(t, f)=\Phi(s, f)=\Psi(s, f) s^{\alpha} \leq K_{1} s^{\alpha}=K_{1}\left(\log t+\frac{\log \frac{\mu}{(\gamma \beta)^{k}}}{k-1}\right)^{\alpha} \leq K_{2}(\log t)^{\alpha+\varepsilon}
$$

and

$$
T(t, g)=\Phi(s, g)=\Psi(s, g) s^{\alpha} \leq K_{3} s^{\alpha}=K_{3}\left(\log t+\frac{\log \frac{\mu}{(\gamma \beta)^{k}}}{k-1}\right)^{\alpha} \leq K_{4}(\log t)^{\alpha+\varepsilon} .
$$

Therefore, we have

$$
T(r, f)=O\left((\log r)^{\alpha+\varepsilon}\right)
$$

and

$$
T(r, g)=O\left((\log r)^{\alpha+\varepsilon}\right)
$$

where

$$
\alpha=\frac{\log 2 M}{\log k}=\frac{\log 2 \sigma}{\log k}+o(1) .
$$

Letting now $\alpha=\frac{\log 2 \sigma}{\log k}$, we obtain the required form. Theorem 2 is proved.

\section{Competing interests}

The author declares that there is no conflict of interests regarding the publication of the article.

\section{Acknowledgements}

The author would like to thank Professor Liangwen Liao and the anonymous referees for their valuable comments and suggestions. The research was supported by NSF of China (11426118), Natural Science Foundation of Jiangsu Province (BK20140767), Natural Science Foundation of the Jiangsu Higher Education Institutions (14KJB110004), Qing Lan Project of Jiangsu Province and Colonel-level topics (JSNU-ZY-01). 


\section{References}

1. Cherry, W, Ye, Z: Nevanlinna's Theory of Value Distribution. Monographs in Math. Springer, Berlin (2001)

2. Hayman, WK: Meromorphic Functions. Clarendon, Oxford (1964)

3. Laine, I: Nevanlinna Theory and Complex Differential Equations. de Gruyter, Berlin (1993)

4. Ablowitz, MJ, Halburd, R, Herbst, B: On the extension of the Painlevé property to difference equations. Nonlinearity 13(3), 889-905 (2000)

5. Chen, ZX, Shon, KH: On zeros and fixed points of differences of meromorphic functions. J. Math. Anal. Appl. 344 373-383 (2008)

6. Chiang, YM, Feng, SJ: On the Nevanlinna characteristic of $f(z+\eta)$ and difference equations in the complex plane Ramanujan J. 16(1), 105-129 (2008)

7. Chiang, YM, Feng, SJ: On the growth of logarithmic differences, difference quotients and logarithmic derivatives of meromorphic functions. Trans. Am. Math. Soc. 361(7), 3767-3791 (2009)

8. Halburd, RG, Korhonen, RJ: Meromorphic solutions of difference equations, integrability and the discrete Painlevé equations. J. Phys. A, Math. Theor. 40, R1-R38 (2007)

9. Halburd, RG, Korhonen, RJ: Difference analogue of the lemma on the logarithmic derivative with applications to difference equations. J. Math. Anal. Appl. 314, 477-487 (2006)

10. Heittokangas, J, Korhonen, R, Laine, I, Rieppo, J, Tohge, K: Complex difference equations of Malmquist type. Comput. Methods Funct. Theory 1(1), 27-39 (2001)

11. Huang, ZB, Chen, ZX: Meromorphic solutions of some complex difference equations. Adv. Differ. Equ. 2009, Article ID $982681(2009)$

12. Ishizaki, K, Yanagihara, N: Wiman-Valiron method for difference equations. Nagoya Math. J. 175, 75-102 (2004)

13. Laine, I, Yang, CC: Clunie theorems for difference and $q$-difference polynomials. J. Lond. Math. Soc. 76(2), 556-566 (2007)

14. Laine, I, Yang, CC: Value distribution of difference polynomials. Proc. Jpn. Acad., Ser. A, Math. Sci. 83(8), 148-151 (2007)

15. Laine, I, Rieppo, J, Silvennoinen, H: Remarks on complex difference equations. Comput. Methods Funct. Theory 5(1), 77-88 (2005)

16. Wang, $\mathrm{H}, \mathrm{Xu}, \mathrm{HY}, \mathrm{Liu}, \mathrm{BX}$ : The poles and growth of solutions of systems of complex difference equations. Adv. Differ Equ. 2013, 75 (2013)

17. Zhang, J, Zhang, J: Meromorphic solutions to complex difference and $q$-difference equations of Malmquist type. Electron. J. Differ. Equ. 2014, 16 (2014)

18. Zhang, JJ, Liao, LW: Further extending results of some classes of complex difference and functional equations. Adv. Differ. Equ. 2010, Article ID 404582 (2010)

19. Zhang, JJ, Liao, LW: On Malmquist type theorem of complex difference equations. Houst. J. Math. 39(3), 969-981 (2013)

20. Zheng, XM, Chen, ZX: Some properties of meromorphic solutions of $q$-difference equations. J. Math. Anal. Appl. 361, 472-480 (2010)

21. Gao, LY: Estimates of $\mathrm{N}$-function and $m$-function of meromorphic solutions of systems of complex difference equations. Acta Math. Sci. Ser. B 32(4), 1495-1502 (2012)

22. Mokhon'ko, AZ, Mokhon'ko, VD: Estimates for the Nevanlinna characteristics of some classes of meromorphic functions and their applications to differential equations. Sib. Mat. Zh. 15(6), 1305-1322 (1974)

23. Goldstein, R: Some results on factorisation of meromorphic functions. J. Lond. Math. Soc. 4(2), 357-364 (1971)

\section{Submit your manuscript to a SpringerOpen ${ }^{\circ}$ journal and benefit from:}

$\checkmark$ Convenient online submission

- Rigorous peer review

Immediate publication on acceptance

- Open access: articles freely available online

- High visibility within the field

- Retaining the copyright to your article 